\title{
SPIN-LATTICE RELAXATION BEYOND THE DEBYE APPROXIMATION*
}

\author{
A.M. WITowskI \\ Institute of Experimental Physics, Warsaw University \\ Hoża 69, 00-681 Warszawa, Poland
}

W. BARDYSZEWSKI

Institute of Theoretical Physics, Warsaw University, Hoża 69, 00-681 Warszawa, Poland

Ch. Kutter, P. Wyder

High Magnetic Field Laboratory, Grenoble, MPI FKF \& CNRS, France

\section{B. Witkowska and A. Mycielski}

Institute of Physics, Polish Academy of Sciences

Al. Lotników 32/46, 02-668 Warszawa, Poland

In this paper we present the results of magnetization relaxation in $\mathrm{HgCdMnTe}$ at high magnetic fields. In this mixed crystal the TA phonons have an energy lower than the spin splitting of the $\mathrm{Mn}^{2+}$ ground orbital singlet at about $20 \mathrm{~T}$, which allows to check the effect of phonons with wave vectors from the edges of the Brillouin zone on the spin-lattice relaxation.

PACS numbers: 76.30.Fc, 63.20.Dj

Usually, spin-lattice relaxation (SLR) is studied using the EPR microwave techniques [1]. Therefore even at low (helium) temperatures the energies involved are smaller than or comparable with thermal energy $k T$. On the other hand, these energies are much smaller than the Debye energy, thus phonons taking part in the relaxation process have small wave vectors. High magnetic field investigations of spin-lattice relaxation make it possible to study not only relaxation in pairs (usually not possible at low fields) [2,3], but also relaxation caused by acoustic phonons with wave vectors far from the center of the Brillouin zone (BZ). In this paper we present experimental investigations of SLR due to the interaction of spins with phonons which cannot be described by the Debye approximation.

*This work was partially supported by the State Committee for Scientific Research (Republic of Poland) through grant \#224109203. 
Our experimental method is based on heating the lattice with far infrared (FIR) laser pulses [4]. The spins then warm up to reach thermal equilibrium with the "hot" lattice. Because magnetization in our system is given by the spin alignment (paramagnetic sample), changes of the spin states appear as magnetization changes and are detected with a pick-up coil [4]. Several FIR laser lines were used to ensure that the results are not photon energy dependent. The nonresonant method allows studies of SLR as a function of magnetic field in a broad range of the external magnetic fields (up to $24 \mathrm{~T}$ ).

The SLR investigations at low fields show the effectiveness of long wa velength acoustic phonons (both longitudinal and transverse modes) in the relaxation process. It is not obvious that phonons with very large wave vectors (from edges of BZ) will also be very effective due to the short wavelength and their lower symmetries. HgCdMnTe mixed crystals give a unique opportunity of studying the above mentioned problem. In HgTe crystals the TA phonons have an energy lower than about $2.5 \mathrm{meV}$ [5] (maximum of the TA phonons density of states (PDoS) is at about $2 \mathrm{meV}$ ). In order to have the spin system, magnetic ions should be introduced. We choose the manganese ions because they have an orbital singlet as the ground state. The spin splitting of this state reaches the energy equal to the maximum energy of TA phonons at about $20 \mathrm{~T}$ ( $g$ factor of 2). Since $\mathrm{HgTe}$ with a small admixture of manganese is a zero gap semiconductor, therefore cadmium is added (more than 15 atomic \%) to open the energy gap. An open gap allows the FIR radiation to pass through the crystal, which is essential in our experimental method [3] (homogeneous heating of the lattice). Free carriers can create additional paths of spin-lattice relaxation, therefore their concentration should be as small as possible. This can be achieved by proper annealing of the material ( $\mathrm{HgCdMnTe})$.

The measurements were performed at $2 \mathrm{~K}$ for a crystal with $30 \%$ of cadmium and $1.5 \%$ of manganese, which was n-type with free carrier concentration at this temperature of the order of $10^{15} \mathrm{~cm}^{-3}$. At such temperature one can expect the relaxation due to the direct process (relaxation with emission and absorption of one phonon) which should be therefore strongly affected by the phonon density of states (e.g. [1]). Our results (Fig. 1) show a rapid increase in the relaxation rates (RR), when the splitting becomes comparable with the energy of phonons from the $\mathrm{BZ}$ edge (the peak in the phonon density of states) and later an abrupt reduction of RR for splitting bigger than the maximum of the TA phonon energy. It is also seen that the results do not depend on the wavelength of the FIR laser pulses used to create the thermal inequilibrium between spins and the lattice. The quite large relaxation rates at low fields are probably due to the presence of free carriers. In high magnetic fields (above $14 \mathrm{~T}$ ) the direct relaxation of Mn spins to the lattice becomes the dominant mechanism of the SLR. The relaxation rates given by this mechanism are related to the phonon density of states. Therefore, the magnetic field dependence of the RR reflects structures of the PDoS.

From the presented experimental data it is clearly seen that the TA phonons with wave vectors from the edge of the Brillouin zone play a very important role and are very effective in the spin-lattice relaxation. At high magnetic fields interaction with these phonons creates the dominant spin-lattice relaxation path. 


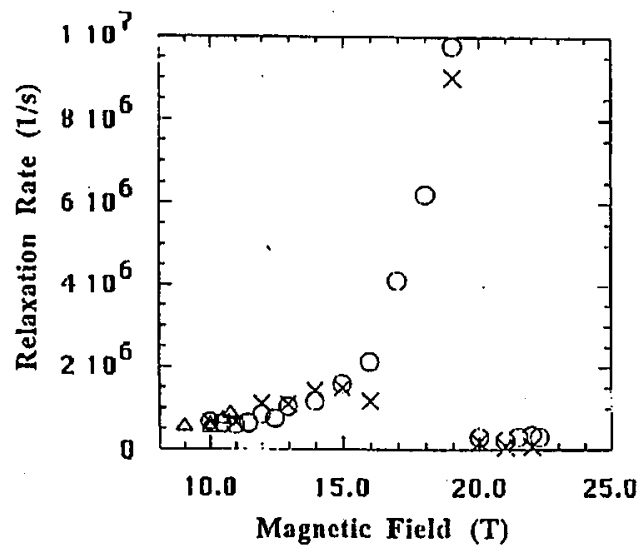

Fig. 1. The relaxation rate as a function of external magnetic field for $\mathrm{Hg}_{0.685} \mathrm{Cd}_{0.3} \mathrm{Mn}_{0.015} \mathrm{Te}$ at temperature $2 \mathrm{~K}$. Different symbols represent data obtained with different FIR laser pulses and different pick-up coils.

\section{Acknowledgment}

One of us (A.M.W.) acknowledges the financial support from the European Community Grant (No. ERB-CIPA-CT-92-0211) for the stay in Grenoble. The authors are grateful to Dr. M.L. Sadowski for a critical reading of the manuscript. We would also like to thank Prof. M. Grynberg for valuable discussions. We are indebted to Dr. W. Dobrowolski and E. Grodzicka for sample characterization.

\section{References}

[1] K.J. Standley, R.A. Vaughan, Electron Spin Relaxation Phenomena in Solids, Adam Hilger, London 1969.

[2] Xiaomei Wang, M. Dahl, D. Heiman, P.A. Wolff, P. Becla, Phys. Rev. B 46, 11216 (1992).

[3] T. Strutz, A.M. Witowski, P. Wyder, Phys. Rev. Lett. 68, 3912 (1992).

[4] T. Strutz, A.M. Witowski, P. Wyder, Rev. Sci. Instrum. 64, 1853 (1993).

[5] H. Kepa, T. Giebultowicz, B. Buras, B. Lebech, K. Clausen, Phys. Scr. 25, 807 (1982). 